\title{
Study on Haze Imaging Model and Image Restoration Schemes for Hazy Remote Sensing Images
}

\author{
N.Ameena Bibi, Dr.C.Vasanthanayaki
}

\begin{abstract}
Remote sensing images taken under hazy conditions have poor visibility of the scene, therefore, haze removal or de-hazing is highly desired. A high-quality haze free image can be recovered by combining the Dark Channel Prior and the haze imaging model. Atmospheric light and transmission medium are estimated based on the Dark Channel Prior. Once the atmospheric light and transmission medium are obtained, scene radiance can be easily estimated. The recovered scene radiance is further refined using restoration filters to remove the artifacts present on the image boundaries. In this paper, the haze imaging model is implemented for single remote sensing images and the effectiveness of haze imaging model is analyzed by varying of the patch size, pixel count $\mathrm{p} \%$ and haze quality parameter $\omega$. To improve the de-hazing performance further, restoration filters such as Gaussian filter, median filter, Wiener filter, bilateral filter and guided filter have been implemented and a performance analysis is carried out. A better performance is obtained for a patch size of $32 \times 32$, the pixel count of $0.05 \%$ and the haze quality parameter $\omega$ as 0.85 . The performance of restoration filters is also analyzed in detail by computing subjective and objective metrics. Experimental results show that the Gaussian filter outperforms well and give visually pleasing haze free images.
\end{abstract}

IndexTerms - Atmospheric light, Dark Channel Prior, Haze imaging model, Restoration filters, Transmission medium.

\section{INTRODUCTION}

The objective of the image restoration is to reconstruct the original image from a degraded image without the knowledge of either the true image or the degradation process. Due to light scattering and absorption by atmospheric particles, images/videos obtained from video surveillance, traffic monitoring, astronomical imaging, medical imaging and remote sensing will have poor visibility. Remote sensing images have been widely used in various fields including agriculture, forestry, hydrology and military with the advantages of rich information, high spatial resolution, and a

Manuscript revised June 10, 2019 and published on July 10, 2019

N.Ameena Bibi, Assistant Professor, Department of Electronics and Communication Engineering, Government College of Technology, Coimbatore, India

Dr.C.Vasanthanayaki, Professor, Department of Electronics and Communication Engineering, Government College of Engineering, Salem, India

doi: $10.32622 /$ ijrat.76201940 stable geometric location. Remote sensing images show the environmental information at terrestrial, atmospheric and ocean phenomenology [1].Remote sensing images are taken at a considerable distance from the earth's surface. During propagation, the incoming energy interacts with the atmosphere which degrades the quality of the received image and this degradation is due to certain atmospheric effects such as haze, fog, smoke, cloud etc. and they differ by visibility distance. The atmospheric contribution is due to scattering by air molecules and aerosols and the effect of the path radiance is to reduce the image contrast leading to image degradation [2]. Increasing hazy weather limits the potential application of remote sensing images and poses a huge challenge for the atmospheric correction of remote sensing images [3].Though it is difficult to obtain haze free image without a reference image, an efficient haze imaging model is developed and implemented to restore the single remote sensing hazy image.

Haze is due to particulate matter and the main sources include smoke, road dust, and other particles emitted directly into the atmosphere, as well as particulate matter formed when gaseous pollutants react in the atmosphere and when these dissolve with vapour and grow into droplets when the humidity exceeds approximately $70 \%$ and causing opaque situation known as haze [4].Burning of fuels from transport sector is one of the air pollutants in urban areas [5].These particles often grow in size as humidity increases and affect the interpretation of remote sensing images. Hazy weather can lead to image color distortion and affect the resolution and the contrast of the observed object. Haze formation is the combination of airlight and attenuation where as attenuation reduces the contrast and airlight increases the whiteness in the scene radiance [6]. Remote sensing images taken under hazy conditions often lack visual vividness and appeal and therefore have poor visibility. Therefore, an effective haze removal method is of great significance for improving the visibility of remote sensing images.

There are different approaches to improve the visibility in hazy images and they are subdivided into three categories such as additional information approaches, multiple image approaches, and single image approaches. The additional image approaches employ scene information to remove haze and recover the realistic colors. Multiple image approaches employ two or more images under different weather conditions to remove haze and hence this requires additional expense or hardware to perform effectively. Single image haze removal techniques use strong assumptions and priors and produce acceptable results, Yet there is the need to restore the image using suitable filters. Single image de-hazing is a challenging problem because only a hazy image is given and also it does not require any additional 
information. Single image de-hazing methods can be classified into two main categories i) image enhancement method based on image processing ii) image restoration based on a physical model. Image enhancement based image processing methods improve the contrast of the image but lose some of the information regarding image and hence image restoration methods are preferred.

He et al proposed a simple and effective haze imaging model [7]. Fattal $\mathrm{R}$ proposed a method to estimate atmospheric light and transmission medium based on assumption that transmission and surface shading are locally correlated [8]. Tien Ying Kuo et al recommended the depth estimation module and color analysis module for better de-hazing. The Depth Estimation module uses a median filter, and adopts the gamma correction to avoid halo artifacts and the color analysis module is based on the gray world assumption [9]. Bingquan Huo et al proposed a water shed segmentation method in order to estimate the atmospheric light and also proposed a method to remove the halo artifacts [10].

Bo Hao Chen et al developed an edge collapse de-hazing algorithm to dynamically repair the transmission map and to achieve better results [11]. Shih chia Huang et al have proposed a novel based visibility restoration approach to effectively solve inadequate haze thickness estimation and alleviate color cast problems [12]. Qingsong Zhu et al have presented a linear modeling to model the scene depth of the hazy image with a supervised learning method, as a result of which the transmission medium can be easily estimated [13]. Xiaoxi Pan et al proposed a deformed haze imaging model to recover remote sensing from the non uniform haze [14]. Xiaoguang Li et al employed an appropriate method to extract the atmospheric light and also proposed a contour preserving estimation to obtain the transmission medium [15].

Single image de-hazing methods have proved to be simple and effective. All the researchers have tested the haze imaging model with fixed patch size, pixel count representing A and haze quality parameter $\omega$. In this paper, a survey was carried out to check the effectiveness of haze imaging model for various patch size, pixel count $\mathrm{p} \%$ representing $\mathrm{A}$, and haze quality parameter and also a comparative analysis of various restoration filters were carried out.

\section{HAZE IMAGING MODEL}

Visibility restoration of hazy images comprises of two stages, the haze imaging model and the refinement phase. A hazy image is formed when there are problems with absorption of light and scattering by atmospheric particles between the sensor and the object. The Haze imaging model is an optical model, and it is used to describe the formation of a hazy image captured in hazy weather condition. The Haze free image obtained from haze imaging model contains artifacts and this is removed using restoration filters in the refinement phase. Fig.1 shows the block diagram of the visibility restoration of hazy images.

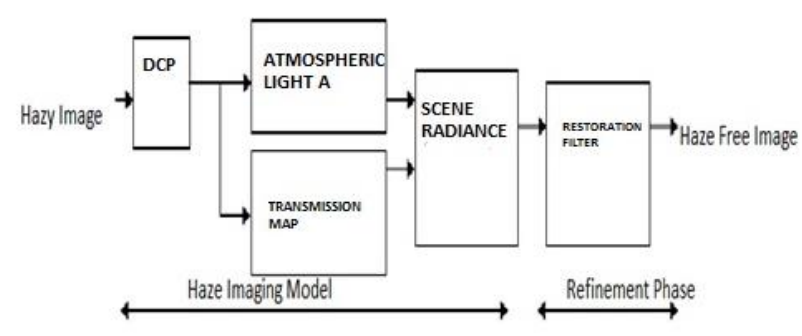

Figure 1: Flow of visibility restoration

Visibility degradation of hazy image is caused by absorption and scattering of light by particles and gases in the atmosphere. The haze imaging model is used to describe the formation of the hazy image and is given as

$$
I(x)=J(x) t(x)+A(1-t(x))
$$

where $\mathrm{I}(\mathrm{x})$ stands for the observed image, $\mathrm{J}(\mathrm{x})$ is the scene radiance, $\mathrm{A}$ is the global atmospheric light and transmission medium $t(x)$ referred to the portion of the light that is not scattered and reaches the sensor. $J(x) t(x)$ is direct attenuation referred to as the portion of light not scattered and reaches the sensor. Fig. 2 shows the pictorial representation of hazy image formation. Hazy image obtained is due to a linear combination of direct transmission and atmospheric light. Therefore $\mathrm{J}(\mathrm{x})$ need to be recovered from the hazy image $\mathrm{I}(\mathrm{x})$

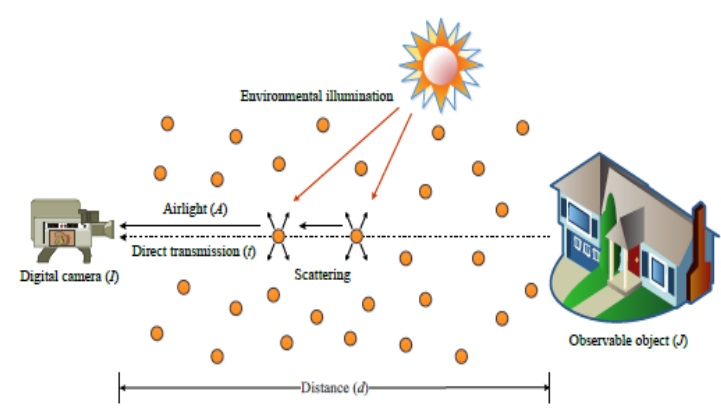

\section{Figure 2:Pictorial representation of Hazy image formation.}

Obviously, it is an ill-posed problem since only $\mathrm{I}(\mathrm{x})$ is given and hence $\mathrm{A}$ and $\mathrm{t}(\mathrm{x})$ need to be estimated. Once $\mathrm{A}$ and $\mathrm{t}(\mathrm{x})$ are estimated, the scene radiance $\mathrm{J}(\mathrm{x})$ can be obtained. In order to estimate Atmospheric light and transmission medium, the Dark Channel Prior is used.

\section{A. Dark channel prior}

Dark Channel Prior proposed by $\mathrm{He}$ at al is based on Blackbody theory [7]. As the black body object in the scene has no reflection, pixel values are very close to zero. But in the hazy image pixel values are non zero which is used to describe the characteristics of atmospheric light in the environment. Therefore intensity of dark channel is a rough approximation of the thickness of haze. The dark channel of a hazy image approximates the haze denseness [16].

$$
\mathbf{J}^{\text {dark }}(\mathbf{x})=\min _{\mathbf{y} \in \Omega(\mathbf{x})}\left(\min _{\mathbf{c} \in\{\mathbf{r}, \mathbf{g , b}\}} \mathbf{J}^{\mathbf{c}}(\mathbf{y})\right)
$$

In order to find the dark channel (DC) for a hazy image 
appropriate patch size should be selected with proper pad size. Selection of Patch size is very important in the DCP. A small local patch is sufficient to estimate the dark channel. which results in reduced calculation time but a small patch size leads to oversaturation and this can be avoided by selecting a large patch size[17]. However selection of a large Patch size leads to the generation of halo effects and block artifacts along the edges of the recovered scene radiance. Image with complicated local textures needs larger local patch size to exclude false textures from the dark channel. Minimum possible patch size that does not produce false textures in the dark channel need to be found for every hazy image by considering application dependent image details.

\section{B. Estimation of atmospheric light}

The air light becomes more dominant as the distance from the object to the observer increases. The haze imaging model indicates that two objects with different radiant energy, located at the same distance from the observer have the same air light[18].The atmospheric light $A_{\infty}$ is best estimated in the most haze-opaque regions and choose the top of $\mathrm{p} \%$ brightest pixels in the dark channel as the most haze-opaque region and the brightest pixel is considered as the global atmospheric light. This approach is more reliable than only searching for the single brightest pixel in the entire image[18]. When a small patch size is used, the pixel for bright objects is considered as candidate pixels yielding inaccurate air light estimation. Therefore the large patch size can prevent selecting of such pixels. Patch size $32 \times 32$ is effective and it is less sensitive to the robustness of any pixel selection $\mathrm{p} \%$.

\section{Estimation of transmission medium}

The transmission describes the portion of light that is not scattered and reaches the sensor, and it is dark in dense haze regions and bright in light haze regions [19]. The transmission depends on two factors: i) the distance between an object and the observer; ii) the scattering coefficient which is related to the turbidity of haze and wavelength and also transmission depends on unknown depths [20].Transmission medium can be defined as

$$
\mathbf{t}(\mathbf{x})=\mathbf{e}^{-\boldsymbol{B d} \mathbf{d}(\mathbf{x})}
$$

$\mathrm{d}(\mathrm{x})$ is the distance between the sensor and observable object $\mathrm{J}(\mathrm{x}), \beta$ is scattering coefficient and in clear weather condition there is no scattering of light and hence $\beta=0$, therefore $t(x)=1$ and the captured image will be the haze free image $\mathrm{I}(\mathrm{x})=\mathrm{J}(\mathrm{x})$. In hazy weather condition, transmission medium $t(x)$ decreases as the scene depth increases, scene radiance is attenuated exponentially with depth. $\mathrm{A}(1-\mathrm{t}(\mathrm{x}))$ is air light which results from scattered light and this leads to shifting of the scene color. When the distance increases, scene depth also increases and when $\mathrm{t}(\mathrm{x})$ is zero, only air light reaches the sensor and captured image contain only air light and hence no scene radiance. $\mathrm{t}(\mathrm{x})$ can also be defined as

$\boldsymbol{t}(\boldsymbol{x})=1-\boldsymbol{\omega} * \min (\min (I(x) / A))$

The results may seem unnatural when the haze is removed completely. Thus, He et al. added a constant $\omega$ within the range of 0 to 1 and it will prevent from under estimation of the transmission medium[7]. A good transmission medium should be constant otherwise halo artifacts will occur in the de-hazed image. Edge information of obtained transmission will be seriously lost, therefore to improve accuracy there is the need to refine the transmission medium.

\section{Scene radiance}

Once $\mathrm{A}$ and $\mathrm{t}(\mathrm{x})$ are estimated, the scene radiance can be computed as

$$
J(x)=\frac{I(x)-A}{t(x)}+A
$$

Recovered scene radiance is prone to noise production when the transmission $\mathrm{t}(\mathrm{x})$ is close to zero and therefore restrict the transmission by lower bound to and it is assigned the value of 0.1

$$
J(x)=\frac{(I(x)-A)}{(\max (t(x), t o))}+A
$$

Incorrect estimation for the transmission medium can lead to some problems such as false texture and blocking artifacts in the recovered scene radiance. Therefore the recovered scene radiance is to be refined using filters.

\section{REFINEMENT PHASE-SCENE RECOVERY BY RESTORATION FILTERS}

The recovered scene radiance $\mathrm{J}(\mathrm{x})$ contains artifacts and this can be removed by restoration filters such as Gaussian, median, Bilateral, wiener, and Guided filter. In this work, implementation and performance of restoration filters are done by computing subjective and objective metrics.

\section{A. Gaussian filter}

The Gaussian filter is a low pass nonlinear filter that smoothens the image. It is useful in removing false color textures, but it unnecessarily blurs the scene radiance when there are no annoying false textures. The Gaussian Filter is effective when proper patch size is used. The 2D Gaussian function is defined as

$$
G(x, y)=\frac{1}{2 \pi \sigma^{2}} e^{-\frac{x^{2}+y^{2}}{2 \sigma^{2}}}
$$

The value of sigma $\sigma$ governs the degree of smoothing, and eventually how the edges are preserved. For the refinement of a hazy image, this filter restores the recovered scene radiance faster and efficiently.

\section{B. Median filter}

The median filter is a well known nonlinear filter which effectively removes noise while preserving edges. Its performance is not much better than a Gaussian blur for a high level of noise. The major limitation of this filter is that fine details will be lost for large window size and also it is a time-consuming process.

\section{Bilateral filter}

Bilateral filter is a nonlinear filter which computes the weighted average of nearby pixels, in a manner very similar to Gaussian convolution. In order to preserve edges while 
smoothing, the bilateral filter takes into account the difference in value with the neighbors The key idea of the bilateral filter is that for a pixel to influence another pixel, it should not only occupy a nearby location but also have a similar value. The bilateral filter is defined as

$$
B F[I p]=\frac{1}{w p} \sum_{q \in S} G_{\sigma s}(|| p-q||) G_{\sigma r}(|| I p-I q||) I q
$$

Where normalization factor

$$
\boldsymbol{w} \boldsymbol{p}=\sum_{q \in S} \boldsymbol{G}_{\sigma s}(|| p-q||) G_{\sigma r}(|| \boldsymbol{I} p-I q||)
$$

$G_{\sigma s}$ is spatial Gaussian weighting that decreases the influence of distant pixels and $G_{\sigma r}$ is range Gaussian that decreases the influence of pixel $q$ which their intensity value differ from Ip. Parameters os and or will specify the amount of filtering for the image I. The bilateral filter is controlled by two parameters $\sigma s$ and or. As the range parameter or increases, the bilateral filter gradually approximates Gaussian more closely and when the spatial parameter os increases, the larger features get smoothened. The disadvantage of Bilateral filter is that it introduces gradient reversal and staircase effect in the restored image.

\section{Wiener filter}

Wiener filter is an optimum filter which minimizes the mean square error. It has the capability of handling both the degradation function as well as noise. The main advantage of Wiener filter is that it is stable in the presence of the nulls. The Wiener filter is used to detect color distortion when image with large white objects is processed using the DCP and this filter is useful in recovering the contrast of large white areas of hazy image[16].

\section{E. Guided filter}

Guided filter is a fast, nonapproximate linear time filter. It has edge preserving, smoothing property like the bilateral filter but does not suffer from gradient reversal artifacts. With the help of the guidance image, it can make the filtering output more structured. The filtering output is computed by

$$
q_{i}=a_{k} I_{i}+b_{k} \forall_{i} \in \omega_{k}
$$

where ak and bk are the average of a and b respectively on the window wk centered at $i$.

$$
\begin{aligned}
& a_{k}=\frac{\frac{1}{\omega} \sum_{i \in \omega_{k}} I_{i} p_{i}-\mu_{k} p_{k}}{\sigma_{k}^{2}+\varepsilon} \\
& b_{k}=p_{k}-a_{k} \mu_{k}
\end{aligned}
$$

where $\mathrm{i}$ is the index of a pixel, and $\mathrm{k}$ is the index of a local square window $\mathrm{w}$ with a radius $\mathrm{r}$, where $\mu_{\mathrm{k}}$ and $\sigma_{\mathrm{k}}$ are the mean and variance of $I$ in the window $k$, and $C$ is a regularization parameter controlling the degree of smoothness. The restored image by guided filter is closer to the real scene. Obviously, transmissions estimated by these methods are darker in dense haze regions than in light haze regions, which is consistent with the haze distribution in a real scene. It has better performance near edges when compared to the bilateral filter[21].

\section{PERFORMANCE ANALYSIS OF THE HAZE IMAGING MODEL}

In this work, experiments were conducted to implement and analyze the haze imaging model and restoration filters to obtain high-quality haze free images. To test the effectiveness of the haze imaging model, atmospheric light, transmission map and elapsed time were analyzed by varying the patch size, the pixel count $\mathrm{p} \%$ representing $\mathrm{A}$ and haze quality parameter $\omega$. The above performance analysis was carried out for 40 input samples and in this paper, results were shown for three input samples Fig. 3a, 3b, 3c are the hazy images used for the analysis of haze imaging model. Atmospheric light, transmission map and elapsed time of haze imaging model are analyzed by varying of the patch size, count representing A and haze quality parameter $\omega$.

\section{A. Effect of patch size}

Selection of patch size plays a significant role in the dark channel prior. Most of the researchers used fixed patch size for estimating the dark channel prior .As atmospheric light mainly depends on the dark channel, the study is carried out for various patch size. Table I. gives the estimated atmospheric light with respect to patch size for two different samples and from results obtained atmospheric light has no significance for a patch size greater than $32 \times 32$ hence a patch size of $32 \times 32$ is acceptable. It is observed that small patch size results in inaccurate atmospheric light estimation because bright pixels are considered as candidate pixels. A good transmission map should be uniform to avoid halo artifacts in a de-hazed image. Fig. 4 shows the effect of patch size on dark channel estimation. Fig. 5a and 5b show transmission map for patch size of $32 \times 32$ and $7 \times 7$ respectively. It is shown that uniform transmission can be obtained for large patch size of $32 \times 32$ and small patch size tends to have color textures.

\section{B. Effect of pixel count $p \%$}

After computing the dark channel, a suitable quantity of bright pixels needs to be computed for atmospheric light estimation. A 400x400 hazy image was considered and study was made with $0.05 \%, 0.1 \%$ and $0.2 \%$. of the total number of pixels. Table II. gives the effect of pixel count $\mathrm{p} \%$ and patch size on atmospheric light estimation. It is found that for large patch size, the dark channel value obtained is small and hence the value of atmospheric light obtained is smaller. Even the $\mathrm{p} \%$ is varied, the variation of atmospheric light is not significant for large patch size. Patch size of $32 \times 32$ and pixel count $\mathrm{p} \%=0.05 \%$ gives better estimation of atmospheric light .

\section{Effect of haze quality parameter $(\omega)$}

If the haze is removed completely, the image seems unnatural due to loss of depth information, hence $\omega$ is considered in transmission medium estimation. In this work, $\omega=0.85$ and $\omega=0.9$ are used for transmission medium estimation. Better estimation of transmission medium can be obtained for $\omega=0.85$. From Fig 6 shows scene radiance for two samples and the image seems natural for $\omega$ value of 0.85 and it results in a good estimation of the transmission 


\section{E-ISSN: 2321-9637}

Available online at www.ijrat.org

medium.

\section{Computational time}

To study the effect of variation in parameters(patch size, the pixel count $\mathrm{p} \%$ representing $\mathrm{A}$ and haze quality parameter $\omega)$ on the complexity of haze imaging model implementation, computational time is calculated and compared in Table III. When patch size increases, elapsed time increases proportionally for the DC (dark channel) and transmission medium. Computation time is constant for atmospheric light and scene radiance for different patch size and its around 0.02 seconds for atmospheric light and 0.018 seconds for scene radiance. Similarly, there is no effect of $\mathrm{p} \%$ on computation time for atmospheric light.

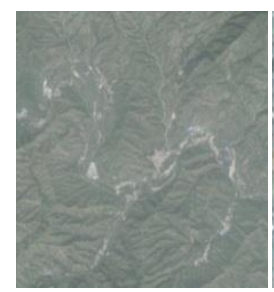

a)sample1

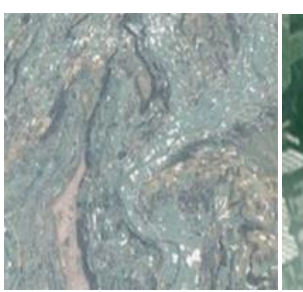

b)sample2

Figure 3: Hazy images

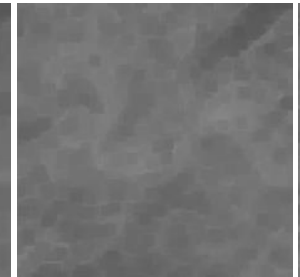

b) $15 \times 15$

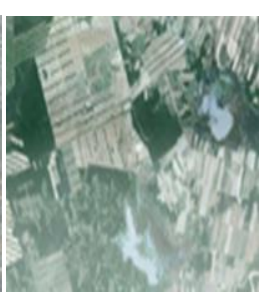

c)sample3

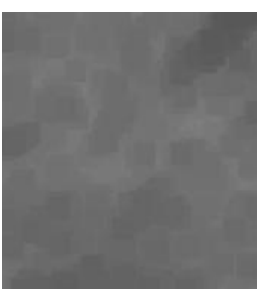

a) $32 \times 32$
Figure 4: Effect of patch size on dark channel estimation
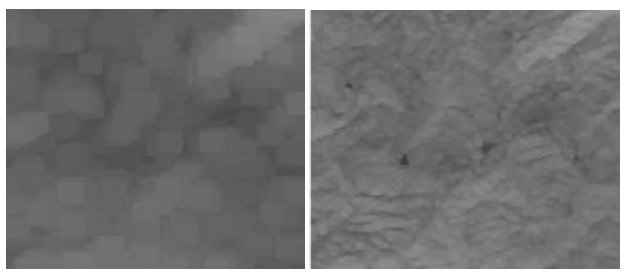

a)patch size $32 \times 32$ b) patch size $7 \times 7$ Figure 5: Transmission map

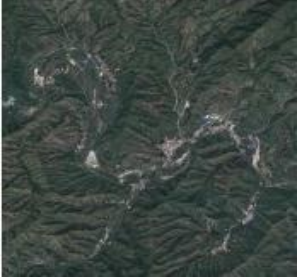

a)

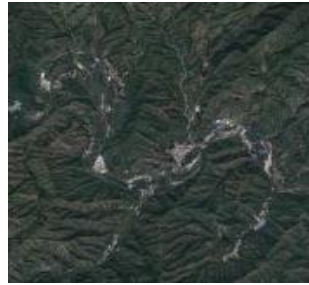

c)

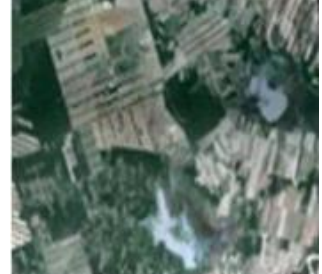

b)

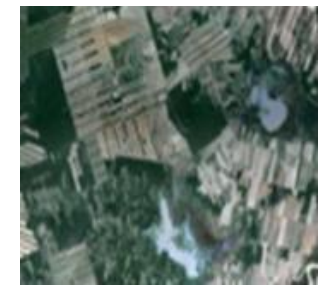

d)
Figure 6: scene radiance for image samples a) and b) for $\omega=0.85$ c) and d) for $\omega=0.9$

Table I Atmospheric light for various patch size

\begin{tabular}{|l|c|c|}
\hline $\begin{array}{l}\text { Patch } \\
\text { Size }\end{array}$ & $\begin{array}{l}\text { Atmospher } \\
\text { ic light } \\
\text { A } \\
\text { (sample1) }\end{array}$ & $\begin{array}{l}\text { Atmospheri } \\
\text { c light A } \\
\text { (sample 2 ) }\end{array}$ \\
\hline $42 \times 42$ & 0.5928 & 0.6275 \\
\hline $40 \times 40$ & 0.5905 & 0.6287 \\
\hline $36 \times 36$ & 0.5925 & 0.627 \\
\hline $32 \times 32$ & 0.5745 & 0.6463 \\
\hline $17 \times 17$ & 0.5847 & 0.8060 \\
\hline $15 \times 15$ & 0.6086 & 0.8188 \\
\hline $11 \times 11$ & 0.6764 & 0.7323 \\
\hline $7 \times 7$ & 0.7169 & 0.746 \\
\hline
\end{tabular}

Table II Comparison of Atmospheric light for various patch size and pixel selection $\mathrm{p} \%$

\begin{tabular}{|c|c|c|c|}
\hline $\begin{array}{c}\text { Pixel } \\
\text { selection } \\
\mathrm{p} \%\end{array}$ & $\begin{array}{c}\text { Atmospheric } \\
\text { light } \\
\text { A for } 7 \times 7\end{array}$ & $\begin{array}{c}\text { Atmospheric } \\
\text { light } \\
\text { A for } \\
15 \times 15\end{array}$ & $\begin{array}{c}\text { Atmospheric } \\
\text { light A } \\
\text { for } 32 \times 32\end{array}$ \\
\hline $0.1 \%$ & 0.7092 & 0.5925 & 0.5984 \\
\hline $0.2 \%$ & 0.6958 & 0.6049 & 0.5840 \\
\hline $0.05 \%$ & 0.7169 & 0.6086 & 0.5745 \\
\hline
\end{tabular}


International Journal of Research in Advent Technology, Vol.7, No.6, June 2019

E-ISSN: 2321-9637

Available online at www.ijrat.org

Table III Comparison of computational time for DC and $\mathbf{t}(\mathbf{x})$ for various parameters

\begin{tabular}{|c|c|c|c|c|c|c|c|c|c|c|c|c|c|c|c|c|c|c|}
\hline & \multicolumn{3}{|c|}{$\begin{array}{l}\omega=0.85, \text { patch } \\
\text { size }=32 \times 32\end{array}$} & \multicolumn{3}{|c|}{$\begin{array}{l}\omega=0.9, \text { patch } \\
\text { size }=32 \times 32\end{array}$} & \multicolumn{3}{|c|}{$\begin{array}{l}\omega=0.85, \text { patch } \\
\text { size }=17 \times 17\end{array}$} & \multicolumn{3}{|c|}{$\begin{array}{l}\omega=0.9, \text { patch } \\
\text { size }=17 \times 17\end{array}$} & \multicolumn{3}{|c|}{$\begin{array}{l}\omega=0.85, \text { patch } \\
\text { size }=15 \times 15\end{array}$} & \multicolumn{3}{|c|}{$\begin{array}{l}\omega=0.9, \text { patch } \\
\text { size }=15 \times 15\end{array}$} \\
\hline $\mathrm{p}$ & 0.1 & 0.2 & .05 & 0.1 & 0.2 & .05 & 0.1 & 0.2 & .05 & 0.1 & 0.2 & .05 & 0.1 & 0.2 & .05 & 0.1 & .02 & .05 \\
\hline$\%$ & $0 \%$ & $\%$ & $\%$ & $0 \%$ & $\%$ & $\%$ & $0 \%$ & $\%$ & $\%$ & $0 \%$ & $\%$ & $\%$ & $0 \%$ & $\%$ & $\%$ & $0 \%$ & $\%$ & $\%$ \\
\hline DC & 6.5 & 4.6 & 4.6 & 4.02 & 5.31 & 4.81 & 4.6 & 3.48 & 4.18 & 4.8 & 3.92 & 3.2 & 4.5 & 3.9 & 3.6 & 4.2 & 4.12 & 4.15 \\
\hline $\mathrm{t}(\mathrm{x})$ & 4.23 & 4.35 & 6.14 & 5.8 & 5.37 & 5.53 & 3.31 & 4.84 & 3.24 & 3.88 & 2.96 & 3.6 & 3.4 & 4.9 & 4.37 & 3.06 & 3.63 & 3.98 \\
\hline
\end{tabular}

Table IV

Relation between relative humidity and correlation coefficient

\begin{tabular}{|l|l|}
\hline $\begin{array}{l}\text { Correlation } \\
\text { Coefficient }\end{array}$ & $\begin{array}{l}\text { Relative } \\
\text { humidity }\end{array}$ \\
\hline 0.95 & below $50 \%$. \\
\hline 0.76. & \begin{tabular}{l} 
50-60\% \\
greater than \\
\hline 0.42 to 0.37
\end{tabular} \\
\hline
\end{tabular}

Table $\mathrm{V}$ performance metrics of various restoration filters

\begin{tabular}{|c|c|c|c|c|c|c|c|c|}
\hline \multicolumn{5}{|c|}{ PATCH SIZE $32 X 32 \mathrm{p} \%=0.1, \omega=0.9$} & \multicolumn{4}{|c|}{ PATCH SIZE 32X32 $\mathrm{p} \%=0.1, \omega=0.85$} \\
\hline $\begin{array}{l}\text { PERFORMAN } \\
\text { CE METRICS }\end{array}$ & $\begin{array}{c}\text { HAZE } \\
\text { DENSITY }\end{array}$ & EPI & $\begin{array}{l}\text { CORRELATI } \\
\text { ON } \\
\text { COEFFICIENT }\end{array}$ & $\mathrm{M}^{\text {SSI }}$ & $\begin{array}{c}\text { HAZE } \\
\text { DENSITY }\end{array}$ & EPI & $\begin{array}{l}\text { CORRELATI } \\
\text { ON } \\
\text { COEFFICIENT }\end{array}$ & $\mathrm{M}^{\mathrm{SSI}}$ \\
\hline $\begin{array}{c}\text { BEFORE } \\
\text { RESTORATION }\end{array}$ & 0.2302 & 0.7051 & 0.7051 & 0.9722 & 0.2659 & 0.7007 & 0.7007 & 0.9757 \\
\hline GAUSSIAN & 0.2302 & 0.996 & 0.996 & 1 & 0.2658 & 0.9959 & 0.9959 & 1 \\
\hline MEDIAN & 0.2297 & 0.9733 & 0.9733 & 1 & 0.2653 & 0.9737 & 0.9737 & 1 \\
\hline BILTERAL & 0.2303 & 0.8975 & 0.8975 & 1 & 0.2659 & 0.899 & 0.899 & 1 \\
\hline WIENER & 0.2304 & 0.9493 & 0.9493 & 1 & 0.266 & 0.9489 & 0.9489 & 1 \\
\hline GUIDED & 0.2303 & 1 & 1 & 1 & 0.2659 & 1 & 1 & 1 \\
\hline \multicolumn{5}{|c|}{ PATCH SIZE $32 X 32 \mathrm{p} \%=0.2, \omega=0.9$} & \multicolumn{4}{|c|}{ PATCH SIZE $32 X 32 \mathrm{p} \%=0.2, \omega=0.85$} \\
\hline $\begin{array}{c}\text { BEFORE } \\
\text { RESTORATION }\end{array}$ & 0.2226 & 0.7052 & 0.7052 & 0.9722 & 0.2592 & 0.7008 & 0.7008 & 0.9747 \\
\hline GAUSSIAN & 0.2225 & 0.9961 & 0.9961 & 1 & 0.2591 & 0.9959 & 0.9959 & 1 \\
\hline MEDIAN & 0.222 & 0.9736 & 0.9736 & 1 & 0.2586 & 0.974 & 0.974 & 1 \\
\hline BILTERAL & 0.2226 & 0.8987 & 0.8987 & 1 & 0.2593 & 0.9001 & 0.9001 & 1 \\
\hline WIENER & 0.2227 & 0.9501 & 0.9501 & 1 & 0.2594 & 0.9496 & 0.9496 & 1 \\
\hline GUIDED & 0.2226 & 1 & 1 & 1 & 0.2593 & 1 & 1 & 1 \\
\hline \multicolumn{5}{|c|}{ PATCH SIZE $32 X 32 \mathrm{p} \%=.05, \omega=0.9$} & \multicolumn{4}{|c|}{ PATCH SIZE $32 X 32 \mathrm{p} \%=.05, \omega=0.85$} \\
\hline $\begin{array}{c}\text { BEFORE } \\
\text { RESTORATION }\end{array}$ & 0.2179 & 0.7043 & 0.7043 & 0.9727 & 0.2551 & 0.6999 & 0.6999 & 0.9752 \\
\hline GAUSSIAN & 0.2178 & 0.9961 & 0.9961 & 1 & 0.255 & 0.996 & 0.996 & 1 \\
\hline MEDIAN & 0.2173 & 0.9738 & 0.9738 & 1 & 0.2545 & 0.9743 & 0.9743 & 1 \\
\hline BILTERAL & 0.2179 & 0.8997 & 0.8997 & 1 & 0.2551 & 0.9011 & 0.9011 & 1 \\
\hline WIENER & 0.218 & 0.9508 & 0.9508 & 1 & 0.2553 & 0.9502 & 0.9502 & 1 \\
\hline GUIDED & 0.2179 & 1 & 1 & 1 & 0.2551 & 1 & 1 & 1 \\
\hline
\end{tabular}




\section{QUANTITATIVE ANALYSIS OF VARIOUS RESTORATION FILTERS}

An efficient restoration filter is to be identified for better refinement of de-hazing. For that the performance of Gaussian, median, wiener, bilateral and guided filter are analyzed with the following performance indicator.

\section{A. Structural Similarity Index(SSIM)}

It measures the structural similarity between input and dehazed images through correlation (s), luminance (l) and contrast(c).

$$
S\left(I_{G S}, I_{F}\right)=\frac{\sigma_{G S, F}}{\sigma_{G S} \sigma_{F}} ; l\left(I_{G S}, I_{F}\right)=\frac{2 \mu_{G S} \mu_{F}}{\mu_{G S}^{2}+\mu_{F}^{2}} ; c\left(I_{G S}, I_{F}\right)=\frac{2 c_{G S} \sigma_{F}}{\sigma_{G S}^{2}+\sigma_{F}^{2}} ;
$$

$$
\operatorname{SSIM}\left(I_{G S}, I_{F}\right)=S\left(I_{G S}, I_{F}\right) * l\left(I_{G S}, I_{F}\right) * c\left(I_{G S}, I_{F}\right)
$$

where IGS,$\mu_{G S}$ and $\sigma_{G S}^{2}$ are the input hazy image, input hazy mean and input hazy image variance, respectively. $\mathrm{I}_{\mathrm{F}}, \mu_{\mathrm{F}}$ and $\sigma_{\mathrm{F}}^{2}$ are the filtered image, filtered image mean and filtered image variance, respectively. $\sigma_{G S F}$ is the covariance between input hazy image and filtered images. After simplifications, the SSIM is given by,

$\operatorname{SSIM}\left(I_{G S}, I_{F}\right)=\frac{\left(2 \mu_{G S} \mu_{F}+C_{1}\right)\left(2 c_{G S} \sigma_{F}+C_{2}\right)}{\left(\mu_{G S}^{2}+\mu_{F}^{2}+C_{1}\right)\left(\sigma_{G S}^{2}+\sigma_{F}^{2}+C_{2}\right)}$

Where $\mathrm{c} 1=0.01 . \mathrm{dr}$ and $\mathrm{c} 2=0.03 . \mathrm{dr}$ with $\mathrm{dr}=255$. Larger values indicate good SSIM.

\section{B. Correlation coefficient}

It is a measure of similarity between original image and the dehazed image. It also used to estimate relative humidity in the hazy image. Relative humidity is the major factor that influences the formation of hazy weather. When relative humidity is below $60 \%$, visibility is highly influenced by concentration of particulate matter. Relation between relative humidity and correlation coefficient is shown in Table IV.

\section{Edge Preservation Index(EPI)}

It measures the edge preservation ability of a filter and it is expressed as

$$
\mathrm{EPI}=\frac{\sum(\Delta y-\overline{\Delta y})(\Delta x-\overline{\Delta x})}{\sum(\Delta y-\overline{\Delta y})^{2}(\Delta x-\overline{\Delta x})^{2}}
$$

Larger value indicates good edge preservation.

\section{Haze density}

Haze density of a hazy image is obtained by convolving the hazy image with the smoothing function. Then, the haze veil is generated by the means of $\mathrm{L}(\mathrm{x}, \mathrm{y})$ [22]. The computation process of the haze veil can also be expressed as follows:

$$
L(x, y)=I(x, y) . F(x, y)
$$

A smaller value implies that the restored image has less haze density.

To study the effect of variation in parameters (patch size, pixel count $\mathrm{p} \%$ representing A and haze quality parameter $\omega$ ) on the complexity of haze imaging model implementation, the computation time is calculated and compared in Table III Performance metrics of various filters such as the Gaussian filter, median filter, Wiener filter, bilateral filter and guided filter are shown in Table V. and Computational time for the various filters is shown in Table VI. A hazy image of size 400x400 with haze density of 0.5249 is considered.

From the performance metrics obtained the Guided and the Gaussian filters restore the image well when compared to other filters. Thus the Gaussian filter can be preferred for restoring the hazy image when compared to the guided filter because of three reasons i) computation time of guided filter is twice that of Gaussian filter ii) Guided filter process on a grayscale image and output obtained by guided is not visually pleasing and seems to be dim when compared with the Gaussian filtered output. iii) The Guided filter also requires an additional information i.e. guidance image.

\section{Table VI Computation time for various filters}

\begin{tabular}{|l|l|}
\hline \multicolumn{2}{|c|}{ Computation time in seconds } \\
\hline GAUSSIAN & 0.2056 \\
\hline MEDIAN & 4.248 \\
\hline BILTERAL & 10.745 \\
\hline WIENER & 0.1803 \\
\hline GUIDED & 0.453 \\
\hline
\end{tabular}

When computational times were compared, the wiener and Gaussian filter compute faster but the Gaussian filter is preferred due to its good performance metrics.

\section{QUALITATIVE EVALUATION}

A input of a hazy image of size 400x400 was considered and analyzed by varying the patch size, pixel selection $\mathrm{p} \%$ and parameter $\omega$. The quality of output image is compared in terms of visual pleasure and haze. Fig. 7 represents the hazy image of size $400 \times 400$ with haze density of 0.5249 .

Fig. 8 shows the various filter output for a patch size of $32 \times 32, p=0.05 \%$ and $\omega=0.85$ and Fig. 9 shows the various filter output for a patch size of $32 \times 32, p=0.05 \%$ and $\omega=0.9$. By comparing both Fig. 8 and 9 better results were obtained for a patch size of $32 \times 32$, pixel percentage $0.05 \%$ and $\omega=0.85$.

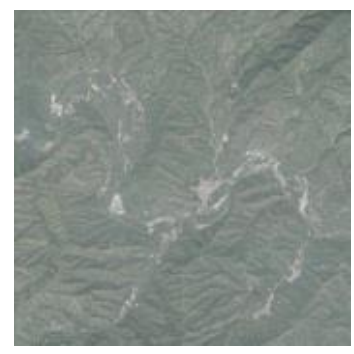

Figure 7: Input hazy image of size $400 \times 400$ 

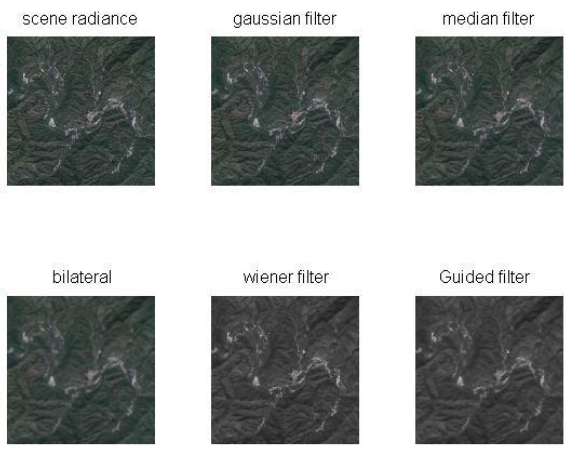

Figure 8 :For a patch size of $32 \times 32, p=.05 \%$ and $\omega=.85$ (a) Haze Free Image before filtering

(b) Gaussian Filtered Image

(c) Median Filtered Image

(d) Bilateral Filtered Image

(e) Wiener Filtered Image

(f) Guided Filtered Image
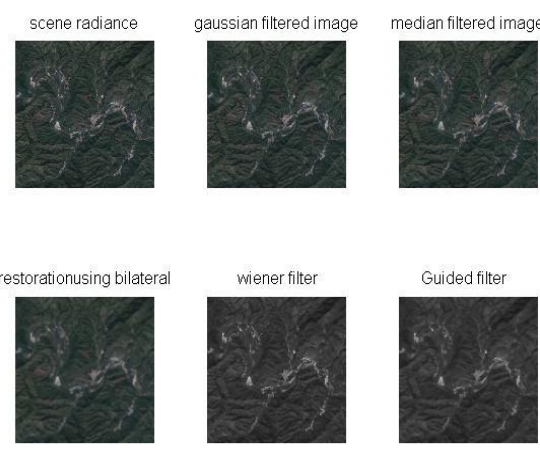

Figure 9: For a patch size of $32 \times 32, p=.05 \%$ and $\omega=.9$

(a) Haze Free Image before Filtering

(b) Gaussian Filtered Image

(c) Median Filtered Image

(d) Bilateral Filtered Image

(e) Wiener Filtered Image

(f) Guided Filtered Image

\section{CONCLUSION}

For the estimation of Atmospheric light, patch size and pixel count $\mathrm{p} \%$ representing A are important. In transmission medium estimation, the patch size and haze quality parameter $\omega$ are important. This paper aims at finding suitable parameters such as patch size, pixel selection $\mathrm{p} \%$ and parameter $\omega$ for effective de-hazing. From the results, it was found that suitable parameters for the haze imaging model are such as patch size of $32 \times 32$, the pixel count of $0.05 \%$ and $\omega=0.85$. While considering the refinement phase ,by using the Gaussian filter, haze is removed completely without sacrificing the fidelity of the colors and all the performance metrics obtained are good especially with low processing time. The Computational time is highly related to the hardware and hence in the future a dedicated hardware can be used to reduce the computational time. We believe that our detailed survey and performance analysis on the haze imaging model and restoration filters will help researchers to have better understanding of the DCP based de-hazing.

\section{REFERENCES}

[1] J. Amanollahi, S. Kaboodvandpour, A. M. Abdullah, M. F. Ramli, "Accuracy assessment of moderate resolution image spectro radiometer products for dust storms in semiarid environment," Int. J. Environ. Sci. Technol., vol.8, pp. 373-380, March 2011.

[2] M.B. Potdar , "Modelling of radiation transfer in earth's hazy atmosphere and calculation of path radiances in IRS LISS-I bands," Journal of the Indian Society of Remote Sensing, vol.18, pp:65-75, 1990.

[3] Zheng Wang, Junshi Xia, Lihui Wang et al. "Atmospheric Correction Methods for GF-1 WFV1 Data in Hazy Weather," Journal of the Indian Society of Remote Sensing, pp: 1-12, April 2017.

[4] L. C. Abdullah, L. I. Wong, M. Saari, A. Salmiaton M. S. Abdul Rashid , "Particulate matter dispersion and haze occurrence potential studies at a local palm oil mill," Int. J. Environ. Sci. Technol, vol. 4, pp. 271-278, March 2007.

[5] Perez Martínez, P.J. Miranda, R.M. Nogueira, et al., "Emission factors of air pollutants from vehicles measured inside road tunnels in São Paulo: case study comparison,” Int. J. Environ. Sci. Technol. ,vol.11, pp: 2155-2168, April 2014.

[6] Yadwinder Singh, Er. Rajan Goyal, "Haze Removal in Color Images Using Hybrid Dark Channel Prior and Bilateral Filter," International Journal on Recent and Innovation Trends in Computing and Communication, vol.2, pp:4165-4171, December 2014.

[7] K M. He, J. Sun, X. Tang, "Single Image Haze Removal Using Dark Channel Prior," IEEE Trans. Pattern Anal. Machine Intell., vol. 33, pp: 2341-2353, December 2011.

[8] 8.R. Fattal, "Single Image Dehazing," Proc. ACM SIGGRAPH, vol.98, pp:1-10, 2008.

[9] Tien Ying Kuo, Yi-chung Lo, "Depth estimation from a monocular view of the outdoors," IEEE Transactions on consumer electronics, vol.57, pp: 817-822, June 2011.

[10] Bingquan Huo, Fengling Yin, "Image dehazing with dark channel prior and novel estimation mode," International Journal of multimedia and Ubiquitous engineering, vol. 10, pp: 13-22, 2015.

[11] Bo Hao Chen, Shih-chia Huang, "Edge collapse-based dehazing algorithm for visibility restoration in real scenes," Journal of display technology, vol:12, pp:964-970, 2015.

[12] Shih Chia Huang, Bo Hao Chen, "An advanced single-image visibility restoration algorithm for real world hazy scenes," IEEE Transactions on industrial engineering, vol. 62, pp:2962-2972, May 2015.

[13] Qingsong Zhu, Jiamimg Mai, Ling Shao, "A fast single image haze removal algorithm using color attenuation prior," IEEE transactions on image processing, vol. 4, pp :3522-3533, 2015.

[14] Xiaoxi Pan, FengyingXie, Zhiguo Jiang, Jihao Yin, "Haze Removal for a Single Remote Sensing Image Based on Deformed Haze Imaging Model," IEEE signal processing letters, vol.22, pp: 1806-1810, 2015.

[15] Xiaoguang Li , Huiying Huang, "An Improved Haze Removal Algorithm Based on Genetic Fuzzy Clustering," International Journal of Hybrid Information Technology, vol. 8, pp:261-270, 2015.

[16] Harpoonamdeep Kaur, Dr. Rajiv Mahajan, "A Review on Various Visibility Restoration Techniques," International Journal of Advanced Research in Computer and Communication Engineering, vol.3, pp : 6622-6625, May 2014

[17] Sungmin Lee, Seokmin Yun, Ju-Hun Nam, Chee Sun Won, Seung-Won Jung, "A review on dark channel prior based image dehazing algorithms," EURASIP Journal on Image and Video Processing, vol.4, pp:1-23, 2016.

[18] Jiao Long, Zhenwei Shi, Wei Tang, Changshui Zhang, "Single remote sensing image dehazing," IEEE. Geosci. Remote Sens. Letters, vol.11, pp:59-63, January 2014.

[19] A. Elisee, Zhengning Wang, Liping Li, "A comprehensive study on fast image dehazing techniques" International Journal of Computer Science and mobile computing, vol.2, pp :146-152, September 2013. 
[20] Bolun Cai, Xiangmin Xu, Kui Jia, Chunmei Qing, Dacheng Tao, “ Dehaze net :An end to end system for single image haze removal," IEEE Transactions on Image Processing, vol. 25, pp:5187-5198, May 2016.

[21] Chieh Chi Kao, Jui Hsin Lai, Shao-Yi Chien, "VLSI Architecture Design of Guided Filter for 30fps full HD video," IEEE Transactions on Circuits and systems for videos, vol:24, pp:513-524, March 2014.

[22] Guo Fan, Tang Jin, Cai Zi-xing, "Objective measurement for image defogging algorithms," J. Cent. South Univ., vol.21, pp: 272-286, 2014.

\section{AUTHORS PROFILE}

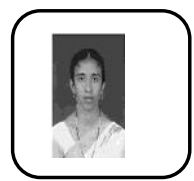

N.Ameena Bibi is a Assistant Professor of Electronics and Communication Engineering at Government College of Technology, Coimbatore. Her work focuses specifically on Dehazing and their impact on the remote sensing and real world images.

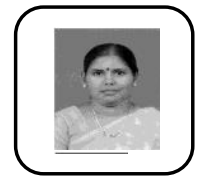

Dr.C.Vasanthanayaki is a Professor \& Head of Department of Electronics and Communication Engineering at Government College of Engineering, Salem. Her work focuses specifically on Image Processing and VLSI Systems 